\title{
Wirken hohe Franchisen kostendämpfend?
}

\author{
Christian Schmida, Konstantin Beck ${ }^{b}$
}

${ }^{a}$ PhD, CSS Institut für empirische Gesundheitsökonomie, Luzern; ${ }^{b}$ Prof. Dr., CSS Institut für empirische Gesundheitsökonomie, Luzern, und Universität Zürich

Das Bundesamt für Gesundheit erwägt eine Anpassung der Franchisenstufen. Es gehe um eine Reduktion der grossen Anzahl an Einzelprämien. In Wirklichkeit geht es wohl darum, die höchsten Franchisenstufen abzuschaffen, um die Solidarität in der sozialen Krankenversicherung zu stärken. Wir zeigen aber, dass eine Reduktion der Wahlfranchisen auch diejenigen schädigt, die von der stärkeren Solidarität profitieren sollten.

\section{Einleitung}

Kürzlich gab das Bundesamt für Gesundheit (BAG) bekannt, dass eine Anpassung der Franchisenstufen ins Auge gefasst wird. Vordergründig geht es dabei um eine Vereinfachung der Krankenversicherung, also um eine Reduktion der grossen Anzahl an Einzelprämien. In Wirklichkeit zielt das BAG wohl eher darauf ab, die höchsten Franchisenstufen abzuschaffen. Es wird nämlich befürchtet, dass gesunde Versicherte die Wahlfranchise hauptsächlich zum Prämiensparen nutzen, und nur teilweise echte Einsparungen durch Konsumverzicht resultieren. Gemäss BAG könne dies zu einer Schwächung der Solidarität in der sozialen Krankenversicherung führen [1] $]^{*}$. Im Folgenden gehen wir deshalb auf die zwei wichtigsten Effekte der Wahlfranchise ein und erläutern, weshalb eine Reduktion der Wahlfranchise kein geeignetes Mittel zur Stärkung der Solidarität in der sozialen Krankenversicherung darstellt.

\section{Wahlfranchisen führen zu Selektion ...}

Die Literatur findet sich unter www.saez.ch $\rightarrow$ Aktuelle Ausgabe oder $\rightarrow$ Archiv $\rightarrow 2015 \rightarrow 35$.

${ }^{* *}$ Im vorliegenden Fall verstehen wir unter Moral Hazard den Effekt, dass Individuen aufgrund der Versicherung mehr Gesundheitsleistungen konsumieren, als sie konsumieren würden, wenn sie alles selber bezahlen müssten.
Tatsächlich führen Wahlfranchisen zu einer Selbstselektion von gesunden Individuen in höhere Franchisenstufen. Dies lässt sich beispielsweise anhand des Wechselverhaltens von Individuen mit einer Franchise von 230 Franken im Jahr 1995 illustrieren [2]. Kosteten Versicherte, die ihre Franchise für das Jahr 1996 nicht erhöht hatten, im Jahr vor dem möglichen Wechsel durchschnittlich 2200 Franken, belaufen sich die vergleichbaren Kosten in der Gruppe der Wechsler auf durchschnittlich lediglich 1377 Franken. Auch sämtliche nachfolgenden Studien weisen auf einen Selbst-
selektions-Effekt hin. Grundsätzlich sollte der Teil des Kostenunterschieds in hohen Franchisenstufen, der durch die Selbstselektion zustande kommt, nicht zu einer Prämienreduktion führen, da die Prämie eben gerade nicht gesundheitsabhängig sein soll. Der Gesetzgeber wirkt deshalb der potentiellen Entsolidarisierung auf zwei Arten entgegen: einerseits indem er den Prämienrabatt beschränkt sowie einen Maximalrabatt festlegt, und andererseits indem der Risikoausgleich einen Teil des Selektionseffekts kompensiert. Konkret werden über den Risikoausgleich etwa 3,2 Milliarden Franken von den Franchisen ab 1000 Franken an die beiden tiefsten Franchisenstufen umverteilt. Der Risikoausgleich neutralisiert dadurch Selektionseffekte und schränkt die Möglichkeit ein, den Versicherten in den Wahlfranchiseprodukten ungerechtfertigt hohe Rabatte zu gewähren. Hinsichtlich der Neutralisierung von Selektionseffekten ist zudem erwähnenswert, dass Politik und BAG hier substantielle Fortschritte machen und machen werden. Der Risikoausgleich wird in den kommenden Jahren - dank dem klaren Revisionsentscheid des Bundesparlaments - noch einmal verschärft [3, 4]. Dies dürfte zu einer weiteren Reduktion von unerwünschten Selektionseffekten führen.

\section{... und zur Nachfragereduktion}

Trotzdem bleibt die Frage, ob Wahlfranchisen zu echten Kosteneinsparungen führen, d.h., ob Moral Hazard** wirklich eine Rolle spielt. Erste Indizien zur Beantwortung dieser Frage liefern die Ergebnisse des RAND Health Insurance Experiment, das in den USA durchgeführt wurde und Spareffekte zwischen 25 und 30\% nach- 
Tabelle 1: Kosteneinsparung durch Wahlfranchisen.

\begin{tabular}{lcccc} 
Franchise & Anteil 2013 & Anzahl Erwachsene & $\begin{array}{l}\text { Kostenreduktion } \\
\text { pro Person }\end{array}$ & $\begin{array}{l}\text { Kostenreduktion } \\
\text { (in 1000 CHF) }\end{array}$ \\
\hline 300 & $43,7 \%$ & 2853426 & 0 & - \\
\hline 500 & $23,7 \%$ & 1547630 & 250 & 386908 \\
\hline 1000 & $4,0 \%$ & 261203 & 322 & 84107 \\
\hline 1500 & $11,7 \%$ & 764020 & 322 & 246014 \\
\hline 2000 & $1,8 \%$ & 117542 & 322 & 37849 \\
\hline 2500 & $15,1 \%$ & 986043 & 322 & $\mathbf{1 0 7 2 3 8 4}$ \\
\hline Summe & & $\mathbf{6 5 2 9 8 6 4}$ & &
\end{tabular}

Bemerkungen: Berechnung der echten Kosteneinsparung (Moral Hazard) aufgrund der Wahlfranchisen unter Verwendung der Versichertenverteilung von 2013 und basierend auf Trottmann, Zweifel und Beck [7].

\footnotetext{
Korrespondenz:

Christian Schmid Institut für empirische Gesundheitsökonomie Tribschenstrasse 21 CH-6002 Luzern christian.schmid[at] css-institut.ch
}

weist. Da die Kostenbeteiligung für die Versicherungsnehmer im Experiment zufällig war, sind hier Selektionseffekte nahezu ausgeschlossen [5]. Somit bilden die Ergebnisse einen Richtwert, lassen sich aber nicht ohne weiteres auf andere Länder übertragen.

Es lässt sich festhalten, dass die Wahlfranchisen in der Schweiz zu echten Kosteneinsparungen führen.

Für die Schweiz gibt es (mindestens) zehn wissenschaftliche Untersuchungen zum Moral-Hazard-Effekt, wobei neun dieser zehn zum Schluss kommen, dass ein echter Einspareffekt existiert. Wilhelm [6] weist beispielsweise einen Effekt von 12\% für mittlere und 27\% für hohe Wahlfranchisen nach. Diese Ergebnisse werden durch Gardiol, Geoffard und Grandchamp [7] sowie Van Kleef, Beck und Buchner [8] bestätigt, die, abhängig von der Wahlfranchise, jährliche Einsparungen von bis zu 697 Franken beziehungsweise 443 Franken ermitteln. Untersuchungen, die nach der letzten Erhöhung der Maximalfranchise gemacht wurden, kommen zu ähnlichen Ergebnissen. Trottmann, Zweifel und Beck [9] finden Einsparungen von 250 Franken bei Franchise 500 und 322 Franken ab Franchise 1000, und Gerfin, Kaiser und Schmid [10] schätzen den Moral-Hazard-Effekt für die drei höchsten Wahlfranchisen auf über 25\%. Die einzige Studie, die keinen Effekt nachweist, wurde kurz darauf durch den gleichen Autor verworfen [11, 12]. Zusammenfassend lässt sich also festhalten, dass die Wahlfranchisen in der Schweiz zu echten Kosteneinsparungen führen. Insbesondere zeigt sich, dass die Reduktion des Moral-Hazard-Effekts, sofern er nachgewiesen wird, ganz sicher in den höchsten Franchisenstufen wirkt.

\section{Gesamteffekt auf die Kosten und Prämien}

Da Moral Hazard offensichtlich existiert, würde eine Reduktion der Wahlfranchisen zu einer erhöhten Nachfrage führen, welche die Kosten und damit die Prämien beeinflusst. Im Gegensatz dazu ist der reine Selektionseffekt kostenneutral. Er führt lediglich dazu, dass die Prämienlast umverteilt wird. Entsprechend dürfen wir bei der Beurteilung des Gesamteffekts nur den Moral-Hazard-Effekt berücksichtigen. Unter Verwendung der Versichertenverteilung von 2013 und der Einsparungen nach Trottmann, Zweifel und Beck [9] erhalten wir einen Spareffekt von 1,1 Milliarden Franken (Tab. 1) oder 5\% der Nettoleistungen der Erwachsenen. Anders ausgedrückt: Eine Reduktion der Wahlfranchisen führt nicht zu tieferen Prämien, sondern zu einem Prämienanstieg von durchschnittlich rund 5\%. Da wir tiefe Werte für den Moral Hazard annehmen, ist letzterer vorsichtig geschätzt.

\section{Fazit}

Das Vorliegen eines Kosteneffekts bei Wahlfranchisen ist in der Literatur zur Schweiz unumstritten, einzig die Stärke des Effekts differiert bei den verschiedenen Studien. Ebenso existiert ein unerwünschter Selektionseffekt, der jedoch kostenneutral ist und durch geeignete Massnahmen reduziert werden kann. Der Risikoausgleich stellt hier ein sehr wirkungsvolles Instrument dar, um ungerechtfertigte Rabatte zu verhindern und Selektionseffekte zu neutralisieren. Eine Senkung oder gar die Abschaffung der Wahlfranchisen gehört jedoch nicht zu diesen Massnahmen, da die Kosten und somit auch die Prämien dadurch um rund 1,1 Milliarden oder

\section{Eine Reduktion der Wahlfranchisen würde zu} einer erhöhten Nachfrage führen, welche die Kosten und damit die Prämien beeinflusst.

5\% steigen könnten. Wenn nur ein Teil des Kostenanstiegs über die nun höhere Prämie der Individuen mit vormals hohen Franchisen bezahlt wird, müssten die anderen Versicherten den Rest finanzieren. Eine Reduktion der Wahlfranchisen schädigt auch diejenigen, die von der stärkeren Solidarität profitieren sollten, und ist daher kaum ein geeignetes Mittel, um die Solidarität in der sozialen Krankenversicherung zu stärken.

\section{Disclosure statement}

Die Autoren danken der CSS Versicherung AG für die Unterstützung dieser Analyse. Die hier geäusserte Meinung ist die der Autoren und deckt sich nicht zwingend mit der Meinung der CSS Gruppe. 


\section{Literatur}

1 BAG. 413.1 Factsheet Weniger Franchisenstufen; 2015. [Online]. www.bag.admin.ch/gesundheit2020/14638/14672/index. html?lang=de.

2 Beck K, Engler N, Keller B. Praxis des Risikomanagements: Prämien und Kostenbeteiligung. In: Beck K (Hrsg.). Risiko Krankenversicherung: Risikomanagement in einem regulierten Krankenversicherungsmarkt, 3. Aufl. Bern: Haupt; 2013. S. 143-73.

3 Pirktl L. Verfeinerung des Risikoausgleichs durch Berücksichtigung der Arzneimittelkosten. Soziale Sicherheit CHSS. 1/2015; S. 42-6.

4 Trottmann M, Telser H, Stämpfli D, Hersberger KE, Matter K, Schwenkglenks M. Übertragung der niederländischen PCG auf Schweizer Verhältnisse. Bundesamt für Gesundheit. Bern; 2015.

5 Newhouse JP. Free for all? Lessons from the RAND Health Insurance Experiment. Cambridge, Massachusetts: Harvard University Press 1993.

6 Wilhelm S. Auswirkungen von Wahlfranchisen: Reduktion des Moral Hazard oder bloss Risikoselektion? Masterarbeit. Universität Bern; 2005.
7 Gardiol L, Geoffard PY, Grandchamp C. Separating Selection and Incentive Effects: An Econometric Study of Swiss Health Insurance Claims Data. PSE Working Papers. 2005/38.

8 Van Kleef RC, Beck K, Buchner F. How Self-selection Affects Risk Equalization: The Example of Voluntary Deductibles. In: Van Kleef RC (Hrsg.). Voluntary Deductibles and Risk Equalization A Complex Interaction. Rotterdam: Optima Grafische Communicatie; 2008. S. 43-64.

9 Trottmann M, Zweifel P, Beck K. Supply-side and Demand-side Cost Sharing in Deregulated Social Health Insurance: Which is More Effective? Journal of Health Economics. 2012; S. 231-42.

10 Gerfin M, Kaiser B, Schmid C. Health Care Demand in the Presence of Discrete Price Changes. Health Economics; 2015.

11 Schellhorn M. Wählbare Selbstbehalte in der Krankenversicherung der Schweiz: Nachfragesteuerung oder Selektion? Universität Bern; 2004

12 Gerfin M, Schellhorn M. Nonparametric Bounds on the Effect of Deductibles in Health Care Insurance on Doctor Visits Swiss Evidence. Health Economics. 2006; S. 1011-20. 